\title{
Entropic Bell Inequalities
}

\author{
N. J. Cerf ${ }^{1}$ and C. Adami ${ }^{1,2}$ \\ ${ }^{1}$ W. K. Kellogg Radiation Laboratory and ${ }^{2}$ Computation and Neural Systems \\ California Institute of Technology, Pasadena, California 91125, USA
}

(29 August 1996)

\begin{abstract}
We derive entropic Bell inequalities from considering entropy Venn diagrams. These entropic inequalities, akin to the Braunstein-Caves inequalities, are violated for a quantum mechanical EinsteinPodolsky-Rosen pair, which implies that the conditional entropies of Bell variables must be negative in this case. This suggests that the satisfaction of entropic Bell inequalities is equivalent to the non-negativity of conditional entropies as a necessary condition for separability.
\end{abstract}

PACS numbers: 03.65.Bz,05.30.-d,89.70.+c

The essence of Bell inequalities [1,2] is related to Einstein's notion of "realism" [3]: that an object has "objective properties" whether they are measured or not. Bell inequalities, in their simplest form, reflect constraints on the statistics of any three local properties of a collection of objects. These constraints must be obeyed if the three properties can be independently known for each object. An intuitive discussion of Bell inequalities in this context is due to Wigner 四, D. Consider a set of objects, each characterized by three two-valued (or dichotomic) properties $a, b$, and $c$. Then, grouping the objects as a function of two (out of the three) properties (for instance grouping together objects having property $a$ but not $b$ ), it is easy to build a simple inequality relating the number of objects in various groups defined by different pairs of properties. For example,

$$
n(a, \operatorname{not} b) \leq n(a, \operatorname{not} c)+n(\operatorname{not} b, c) .
$$

While such an inequality only refers to the simultaneous specification of any pair of properties, its satisfaction depends on the existence of a probability distribution for all three. Thus, even when the three properties can not be accessed at the same time (for whatever reason), Eq. (11) still holds provided that there exists such an objective description of each object using three parameters $a, b$, and $c$; therefore, Eq. (11) provides a straightforward test of "local realism" (i.e., the combination of objectivity and locality). As confirmed experimentally [6], an inequality such as Eq. (11) can be violated in quantum mechanics. It is the uncertainty principle (implying that the simultaneous perfect knowledge of two conjugate observables is impossible) which is at the root of such a violation. Arguments similar to those above are used to derive the Bell inequalities [1] or their generalization, the ClauserHorne-Shimony-Holt (CHSH) inequalities [7], and their violation can be traced back to the nonexistence of an underlying joint probability distribution involving incompatible variables.

The purpose of this paper is to show that the violation of Bell inequalities in quantum mechanics is directly connected to the existence of negative quantum entropies, a feature which is classically forbidden. We
KRL preprint MAP-205

have shown in previous work [8] that a consistent quantum information theory treating classical correlation and quantum entanglement on the same footing implies that conditional entropies can be negative. This purely quantum behavior can be traced back to the fact that the eigenvalues of a "conditional density matrix" can exceed one. (In contrast, the conditional probabilities in classical information theory are always bounded by one, which implies the classical property that conditional entropies are non-negative.) Negative conditional entropies appear precisely in the case of quantum entanglement [8], for instance for an Einstein-Podolsky-Rosen (EPR) wavefunction, which is the typical object of Bell-type experiments. As a consequence, it is natural to seek for a relation between this non-classical feature and the violation of Bell inequalities, the standard evidence for the existence of quantum nonlocal correlations. To begin with, we derive an entropic Bell inequality that resembles the conventional one but involves mutual entropies rather than correlation coefficients. This entropic Bell inequality is related to the Braunstein-Caves information Bell inequality [9] and implies Schumacher's triangle inequality for information distances [10]. Unlike those, however, our inequality has a structure isomorphic to the conventional one, and has a simple geometric interpretation based on the ternary entropy diagram describing the Bell variables $a, b$, and $c$. Indeed, we show that the violation of our entropic Bell inequalities implies that one out of three conditional entropies describing $a b c$ must be negative, a feature that eliminates any classical description of the system. We show that these entropic Bell inequalities are violated when performing Bell-type measurements on EPR pairs, for example, but not necessarily at the same angles as the conventional Bell inequalities. Therefore, our entropic Bell inequalities provide another necessary condition for separability, distinct from the standard Bell inequalities.

Consider two widely separated entangled systems in general, or, more specifically, a pair of spin- $1 / 2$ particles in a singlet state (Bohm's 11] version of an EPR pair)

$$
|\Psi\rangle=\frac{1}{\sqrt{2}}(|\uparrow \downarrow\rangle-|\downarrow \uparrow\rangle) .
$$


Assume an observer, acting independently on each particle, can measure the spin component of that particle along two possible orientations, for example with a SternGerlach setup. Let the first observer either measure the $z$-component of one of the particles (and call this observable $A$ and the outcome of the measurement $a$ ) or else the component along an axis making an angle $\theta$ with the $z$-axis (observable $B$, with outcome $b$ ). Correspondingly, the second observer measures (on the second particle) either the $z$-component (observable $A^{\prime}$ ) or else the component making an angle $\phi$ with the $z$-axis (observable C) [12]. Locality implies that the two distant observers have no influence on each other, i.e., the decision to make one of the two possible measurements on the first particle does not affect the outcome of the measurement on the other particle. Indeed, it is known that the marginal statistics of the outcome of the spin measurement on the second particle, $c$ for instance, is unchanged whether one measures $A$ or $B$ on the first particle. Let us now outline a general derivation of conventional Bell inequalities (see, e.g., 13]). Consider 3 dichotomic random variables $A, B$, and $C$ that represent properties of the system and can only take on the values +1 or -1 with equal probability $(1 / 2)$. For our purposes, they stand of course for the measured spin components (either up or down along the chosen axis), i.e., the Bell variables. (As $A^{\prime}$ is fully anticorrelated with $A$, we do not make use of it.) Any random set of outcomes $a, b$, and $c$ must obey

$$
a b+a c-b c \leq 1
$$

along with the two corresponding equations obtained by cyclic permutation $(a \rightarrow b \rightarrow c)$. Indeed, the left-handside of Eq. (3) is equal to 1 when $a=b$, while it is equal to $-1 \pm 2$ when $a=-b$. Taking the average of Eq. (3) and its permutations yields the three basic Bell inequalities

$$
\begin{array}{r}
\langle a b\rangle+\langle a c\rangle-\langle b c\rangle \leq 1 \\
\langle a b\rangle-\langle a c\rangle+\langle b c\rangle \leq 1 \\
-\langle a b\rangle+\langle a c\rangle+\langle b c\rangle \leq 1
\end{array}
$$

relating the correlation coefficients between pairs of variables. Eqs. (5) and (6) can be summarized in the form of the standard Bell inequality 13

$$
|\langle a b\rangle-\langle a c\rangle|+\langle b c\rangle \leq 1 .
$$

The important point is that inequalities (46) involve only the simultaneous specification of two (out of the three) random variables, although it is assumed that the three variables possess an element of reality, i.e., they can in principle be known at the same time, even if not in practice. In other words, it is assumed that there exists an underlying joint probability distribution $p(a, b, c)$, in which case the Bell inequalities (which depend only on the marginal probability $p(a, b)=\sum_{c} p(a, b, c)$ and cyclic permutations) must be satisfied. Therefore, the violation of any of the inequalities (4-6) implies that $a, b$, and $c$ cannot derive from a joint distribution (i.e., cannot be described by any local hidden-variable theory), as emphasized in Ref. [9]. In the following, we will show that the violation of Bell inequalities, while ruling out such a classical underlying description of local realism, still does not contradict a quantum one based on an underlying joint density matrix $\rho_{A B C}$, but forces the corresponding entropies to be negative [8].

Let us derive Bell inequalities akin to the conventional ones, Eqs. (46), but relating entropies for the three dichotomic random variables $A, B$, and $C$. We assume that one has access to the entropy of each variable $H(A)$, $H(B), H(C)$, as well as to the mutual entropy between each pair of variables $H(A: B), H(A: C)$, and $H(B: C)$. Here, the entropies are Shannon entropies [14], given by

$$
H(A)=-\sum_{a} p(a) \log p(a)
$$

and the mutual entropies are defined by

$$
H(A: B)=H(A)+H(B)-H(A B) .
$$

The mutual entropy $H(A: B)$ corresponds to the entropy shared by $A$ and $B$, or in other words to the information about $A$ that is conveyed by $B$ (or conversely). Physically, $H(A: B)$ is closely related to the correlation coefficient between $a$ and $b$. To establish notation, let us also define the conditional entropy $H(A \mid B)$ as the entropy of variable $A$ while "knowing", i.e., having measured, $B$,

$$
H(A \mid B)=H(A B)-H(B),
$$

which allows us to separate any entropy into a conditional and a mutual piece with respect to another variable [15]:

$$
H(A)=H(A \mid B)+H(A: B) .
$$

For a three-variable system we can split information into conditional and mutual information in the same fashion: the information $H(A: B)$, for example, can be split as

$$
H(A: B)=H(A: B \mid C)+H(A: B: C) .
$$

Thus, a conditional information such as $H(A: B \mid C)=$ $H(A C)+H(B C)-H(C)-H(A B C)$ is that piece of an information (between two variables) that is not shared by a third variable, i.e., the information conditional on the third variable. The piece of information that is shared by a third variable can be written as

$$
\begin{aligned}
& H(A: B: C)=H(A)+H(B)+H(C) \\
& \quad-H(A B)-H(A C)-H(B C)+H(A B C) .
\end{aligned}
$$

Let us now construct Bell inequalities involving only informations between pairs of variables (rather than correlation coefficients). Relations between entropies are conveniently represented by entropy Venn diagrams [16, 17], 
and inequalities can easily be read off them. As shown in Fig. 1, the ternary entropy diagram for the Bell variables $A B C$ has $7\left(2^{n}-1\right.$ with $\left.n=3\right)$ entries. We use the symbols $\alpha, \beta, \gamma$ for conditional entropies [e.g., $\alpha=H(A \mid B C)], \bar{\alpha}, \bar{\beta}, \bar{\gamma}$ for conditional informations [e.g., $\bar{\alpha}=H(B: C \mid A)]$, and denote by $\delta=H(A: B: C)$ the mutual information between the three Bell variables. Apart from the marginal statistics of each of the variables $A, B$, and $C$, experimentally we also have access to the the marginal statistics of any pair $(A B, A C$, or $B C)$, yielding six constraints. Consequently, we do not have enough constraints to completely fill in the entropy diagram of Fig. 1: the missing constraint concerns the intrinsic three-body correlation which is not fixed by twobody statistics. The seven entries in the ternary entropy diagram can thus be expressed as a function of the six entropies $H(A), H(B), H(C), H(A: B), H(A: C), H(B: C)$, plus a parameter $\delta$, the inaccessible ternary mutual information.

FIG. 1. Ternary entropy diagram for the Bell variables $A B C$. The entries $\alpha, \bar{\alpha}, \beta, \bar{\beta}, \gamma, \bar{\gamma}$ and $\delta$ are defined in the text. All of them (except $\delta$ ) are non-negative in Shannon information theory 15 .

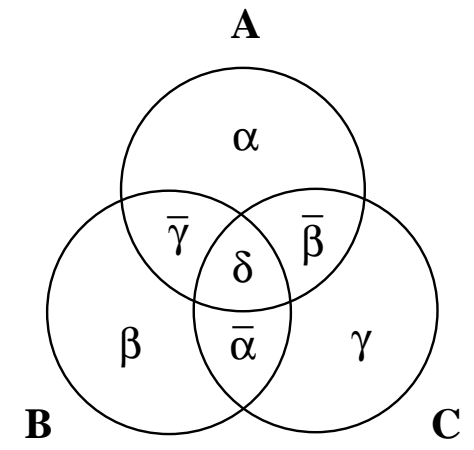

Despite this indeterminacy, the entries can be combined to give expressions independent of $\delta$, and which therefore can be expressed in terms of measurable entropies only. More precisely, we find

$$
\begin{aligned}
\alpha+\bar{\alpha} & =H(A)+H(B: C)-H(A: B)-H(A: C), \\
\beta+\bar{\beta} & =H(B)+H(A: C)-H(A: B)-H(B: C), \\
\gamma+\bar{\gamma} & =H(C)+H(A: B)-H(A: C)-H(B: C) .
\end{aligned}
$$

If $A, B$ and $C$ describe a classical system, it is known that all the entries except $\delta$ are non-negative. Indeed, monotonicity of Shannon entropies implies that conditional entropies such as $\alpha=H(A \mid B C)$ are positive semidefinite [18]. By the same token, conditional informations such as $\bar{\alpha}=H(B: C \mid A)$, as they describe information between two variables when a third is known, are non-negative. (This property is called strong subadditivity 18].) The indeterminacy of $\delta$ can be traced back to the freedom in the choice of a local hidden-variable model to describe the marginal statistics, but its value is unimportant as far as questions of locality are con- cerned. From Eqs. (14 16) it follows straightforwardly that the three inequalities

$$
\begin{aligned}
H(A: B)+H(A: C)-H(B: C) & \leq H(A) \\
H(A: B)-H(A: C)+H(B: C) & \leq H(B) \\
-H(A: B)+H(A: C)+H(B: C) & \leq H(C)
\end{aligned}
$$

must be satisfied if the system $A B C$ is classical. These equations therefore constitute entropic Bell inequalities. Note that in the case where $A, B$, and $C$ have a uniform distribution, one has $H(A)=H(B)=H(C)=1$; the inequalities then become very similar to the standard ones [Eqs. (46)], but relating mutual entropies rather than correlation coefficients. For instance, one can write

$$
|H(A: B)-H(A: C)|+H(B: C) \leq 1
$$

from Eqs. (18) and (19), in perfect analogy with Eq. (77). More generally, the CHSH inequalities for mutual entropies can be derived using the chain rule for entropies. The resulting inequality

$$
H\left(A^{\prime}: B\right)+H(A: C)-H(B: C)+H\left(A: A^{\prime}\right) \leq 2,
$$

is similar in form to the traditional $\mathrm{CHSH}$ inequality (see [13]), and implies the Braunstein-Caves inequality [9] as well as Schumacher's quadrilateral inequality [10.

The converse of the previous reasoning is most interesting. If the data that are extracted from marginal statistics show that one of the three entropic inequalities is violated, it implies that one of the three inequalities $\alpha+\bar{\alpha} \geq 0$ (etc.) is violated. Therefore, since strong subadditivity of quantum entropies [18 implies that $\bar{\alpha}$, $\bar{\beta}$, and $\bar{\gamma}$ are always $\geq 0$, one of the conditional entropies $\alpha, \beta$, or $\gamma$ must be negative, which of course is classically forbidden. Thus, a violation of an entropic Bell inequality always goes hand in hand with the appearance of a negative conditional entropy in Fig. 11. This is the case for example in Bell measurements of EPR pairs, as we show in more detail below. Therefore, negative entropies automatically rule out a description of the system in terms of local hidden variables (or an underlying joint probability distribution). If there cannot be any such description, it is well-known that the system in question is nonseparable [19]. Equivalently, it is shown in Refs. [8, 16] that the concavity of conditional quantum entropies implies that any separable density matrix is characterized by non-negative conditional entropies (see also 20]). In summary, the satisfaction of entropic Bell-inequalities, or equivalently the non-negativity of the corresponding entropies, is a necessary condition for separability, albeit not a sufficient one. Let us show that this condition is distinct from the one based on the satisfaction of traditional Bell inequalities by considering as an example Bell experiments on EPR pairs. In this case, because the outcomes \pm 1 occur with equal probability $(1 / 2)$, the correlation coefficient can be written as $\langle a b\rangle=4 p_{++}-1=1-4 p_{+-}$, 
with $p_{++}\left(p_{+-}\right)$being the probability to observe aligned (anti-aligned) spins. The mutual entropy (in bits) can then trivially be expressed in terms of the corresponding correlation coefficient via

$H(A: B)=\frac{1}{2} \log \left(1-\langle a b\rangle^{2}\right)+\frac{\langle a b\rangle}{2} \log \left(\frac{1+\langle a b\rangle}{1-\langle a b\rangle}\right)$.

Using the standard quantum results for the correlation coefficients, i.e., $\langle a b\rangle=-\left\langle a^{\prime} b\right\rangle=\cos (\theta),\langle a c\rangle=-\cos (\phi)$, and $\langle b c\rangle=-\cos (\theta-\phi)$, we plot in Fig. 2a the left-hand side of Eqs. (17 19) as a function of $\phi$ for the "most violating" angle $\theta=\pi / 3.958$ (the maximum violation occurs at $\phi=\theta / 2)$. Note that the conventional Bell inequalities Eqs. (46) are maximally violated at a different angle $\theta=\pi / 3$. Nevertheless, we have plotted the left-hand side of these equations at the same angle $\theta$ as the entropic ones for comparison in Fig. 2b. Despite the similarity in the structure of the equations, the violation of one conventional Bell inequality does not necessarily imply the violation of an entropic one, or vice versa.

FIG. 2. (a) Left-hand side $L_{E}$ of entropic Bell inequalities Eqs. (17-19) for EPR Bell-measurements with $\theta=\pi / 3.958$. The inequalities are violated if $L_{E}>1$; (b) Left-hand side $L_{C}$ of conventional inequalities Eqs. (4. 6 ) at the same angle.

(a)

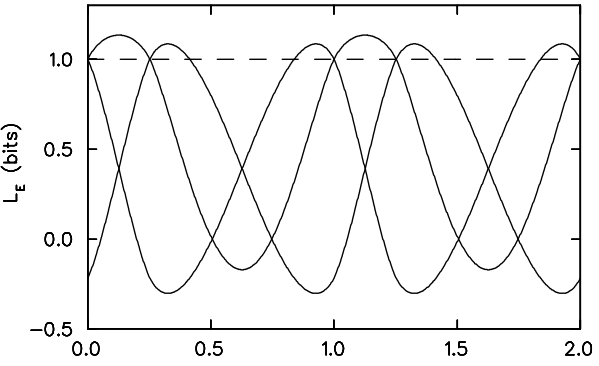

(b)

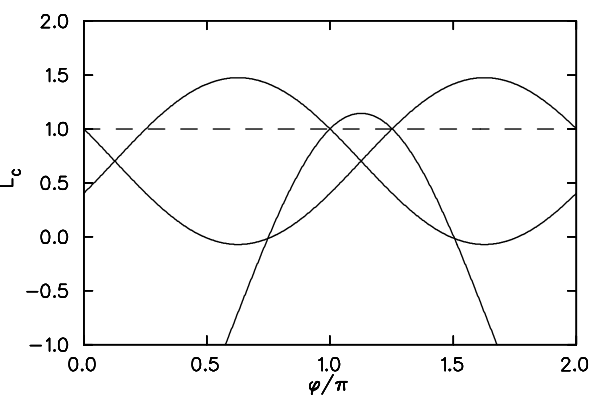

We have derived entropic Bell inequalities by demanding that the conditional entropies arising in the ternary entropy diagram for Bell variables be non-negative, providing a necessary condition for separability. The experimental violation of Bell inequalities, traditionally interpreted as ruling out the existence of a joint probability $p_{a b c}$, therefore also reflects the appearance of negative conditional entropies in Bell-type measurements. In fact, these experiments do not rule out a description in terms of an underlying joint density matrix $\rho_{A B C}$. Yet, the latter does not describe three physical systems as the EPR experiment only involves two detectors. Because of the degree of freedom involved with the choice of $\delta$, such a $\rho_{A B C}$ cannot be constructed explicitly. We are therefore uncertain as to the physical interpretation of $\rho_{A B C}$, a difficulty inherent to independent Bell-type measurements on identically prepared systems. It has recently been suggested that consecutive measurements performed on a single quantum system are more apt at revealing "hidden nonlocality" 21]. It might therefore prove to be fruitful to apply the present analysis to such situations.

This work was supported in part by NSF Grant Nos. PHY 94-12818 and PHY 94-20470.

[1] J. S. Bell, Physics 1, 195 (1964); Rev. Mod. Phys. 38, 447 (1966).

[2] For an introduction and review of Bell inequalities, see e.g. J.F. Clauser and A. Shimony, Rep. Prog. Phys. 41, 1881 (1978); D.M. Greenberger, M. Horne, A. Shimony, and A. Zeilinger, Am. J. Phys. 58, 1131 (1990); D. Harrison, ibid. 50, 811 (1982).

[3] A. Einstein, B. Podolsky, and N. Rosen, Phys. Rev. 47, 777 (1935).

[4] E. P. Wigner, Am. J. Phys. 38, 1005 (1970).

[5] B. d'Espagnat, in: Quantum Theory and Pictures of Reality, ed. W. Schommers, (Springer-Verlag, Berlin, 1989).

[6] A. Aspect, P. Grangier, and G. Roger, Phys. Rev. Lett. 49, 91 (1982); A. Aspect, J. Dalibard, and G. Roger, ibid. 49, 1804 (1982). The experimental refutation of local realism is still controversial due to possible loopholes [see, e.g., E. Santos, Phys. Rev. A 46, 3646 (1992)].

[7] J. F. Clauser, M. A. Horne, A. Shimony, and R. A. Holt, Phys. Rev. Lett. 23, 880 (1969).

[8] N. J. Cerf and C. Adami, e-print quant-ph/9512022.

[9] S. L. Braunstein and C. M. Caves, Phys. Rev. Lett. 61, 662 (1988); Ann. Phys. (N.Y.) 202, 22 (1990).

[10] B. W. Schumacher, Phys. Rev. A 44, 7047 (1991).

[11] D. Bohm, Quantum Theory (Prentice-Hall, Englewood Cliffs, 1951), pp. 611-623.

[12] One of the orientations ( $z$-axis) is the same for both observers; if this constraint is relaxed, one obtains the generalized CHSH inequality [7]. For simplicity, we confine our attention to the standard Bell inequality here.

[13] A. Peres, Quantum Theory: Concepts and Methods, (Kluwer, Dordrecht, 1993).

[14] C.E. Shannon and W. Weaver, The mathematical theory of communication (University of Illinois Press, 1949).

[15] R.B. Ash, Information Theory (Dover, New York, 1965).

[16] N.J. Cerf and C. Adami, e-print quant-ph/9605002.

[17] C. Adami and N.J. Cerf, unpublished (1996).

[18] A. Wehrl, Rev. Mod. Phys. 50, 221 (1978).

[19] R.F. Werner, Phys. Rev. A 40, 4277 (1989).

[20] R. Horodecki and M. Horodecki, Phys. Rev. A 54, 1838 (1996).

[21] S. Popescu, Phys. Rev. Lett. 74, 2619 (1995). 